\title{
Synthetic Polymer
}

National Cancer Institute

\section{Source}

National Cancer Institute. Synthetic Polymer. NCI Thesaurus. Code C82130.

Synthetic high molecular weight molecules made up of a linked series of repeated

monomeric subunits. The monomers can be of the same or different composition. 